\title{
Evidenzbasierte Medizin in der Dermatologie
}

\author{
M. Jung \\ B. Rzany
}

\section{Evidence Based Medicine in Dermatology}

\section{Zusammenfassung}

Im Mittelpunkt der Evidenzbasierten Medizin (EBM) steht der individuelle Patient, für den es gilt, die wirksamste und nebenwirkungsärmste Diagnostik bzw. Therapie zu finden. EBM ist der bewusste Verzicht auf unreflektierte Intuition und die Bevorzugung der Ratio bei der Entscheidungsfindung zur Diagnostik oder Therapie. Die Suche nach dem besten vorhandenen Wissen erfolgt strukturiert nach der Fragestellung, der Suche nach der Information, der Bewertung der gefundenen Quellen, der ärztlichen Entscheidung und deren kritischer Evaluierung. EBM beschränkt sich jedoch nicht nur auf den einzelnen Patienten, sondern bringt sich verstärkt in Entwicklung von Leitlinien ein und dient damit einer kontinuierlichen Qualitätssicherung.

\section{Abstract}

Evidence Based Medicine (EBM) aims at finding the best possible diagnosis or treatment for an individual patient. EBM is the conscious renouncement of unreflected intuition and the preference of ratio in medical decision-making. EBM follows a structured path starting with an answerable question, a systematic literature search, a critical evaluation of the literature found and finally leading to medical decision, which itself needs to be evaluated. EBM is not limited to the individual patients, but is increasingly used in the development of guidelines and represents a base for continuous quality assurance.
Evidenzbasierte Medizin (EBM) hat nichts mit dem im Deutschen üblichen Wort „evident“ zu tun, sondern bezieht sich auf das angelsächische „evidence“ im Sinne einer auf Belegen basierenden Medizin. Evidenzbasierte Medizin (EBM) ist damit der sorgfältige und bewusste Gebrauch der zum gegenwärtigen Zeitpunkt vorhandenen besten wissenschaftlichen Erkenntnisse für medizinische Entscheidungen zur Diagnostik und Therapie individueller Patienten. Dies beinhaltet die Integration der eigenen klinischen Expertise mit der bestmöglichen externen Evidenz aus systematischer Forschung [1]. Evidenzbasierte Dermatologie bedeutet die Umsetzung von EBM bei Patienten mit Hauterkrankungen [2].
Evidenzbasierte Medizin ist an sich nicht neu, wurde jedoch in den 80er Jahren an der McMaster Universität in Kanada in entscheidenden Punkten weiterentwickelt [3]. Im Gegensatz zu den angelsächsischen Ländern befindet sich Evidenzbasierte Dermatologie in Deutschland noch im Stadium des Aufbaus. Die Anzahl der deutschen Dermatologen, die sich in der Cochrane Skin Group oder dem deutschen Cochrane Zentrum engagieren, ist bisher überschaubar. Sichtbares Zeichen für das zunehmende Wissen um die Bedeutung der Evidenzbasierte Medizin ist die Etablierung der ersten Abteilung für Evidenzbasierten Medizin in der Dermatologie an der Charité, die mit Unterstützung des „Stifterverbandes der Deutschen Wissenschaft" geschaffen wurde.

Institutsangaben

Abteilung für Evidenzbasierte Medizin an der Klinik für Dermatologie, Venerologie und Allergologie, Charité Universitätsklinikum, Campus Charité Mitte, Berlin (Direktor: Prof. Dr. W. Sterry)

Widmung

Herrn Prof. Dr. E. G. Jung zum 70. Geburtstag gewidmet.

Korrespondenzadiresse

Prof. Dr. med. B. Rzany · division of evidence based medicine (dEBM) · Klinik für Dermatologie, Venerologie und Allergologie · Universitätsklinikum Charité · Campus Charité Mitte · Schumannstr. 20/23 · 10117 Berlin E-mail: berthold.rzany@charite.de 
Ziel dieser Übersicht ist es, EBM am Beispiel der Dermatologie zu erläutern und, wenn möglich, weiteren Kollegen den Einstieg in die Evidenzbasierte Medizin zu eröffnen.

\section{EBM: Herkunft aus der arithmetischen Medizin}

Die Ursprünge der EBM reichen weit bis ins 18. Jahrhundert zurück, bis zur arithmetischen Medizin, die sich auf Beobachtung und Experiment bezog und die als Vorläufer der klinischen Epidemiologie gilt [4]. Letztere hat sich insbesondere in den 60er Jahren entwickelt und modernisiert. Beispiel dafür ist u.a. die rasant zunehmende und unüberschaubar werdende Zahl von randomisierten klinisch kontrollierten Studien, die letztendlich die wissenschaftliche Grundlage für ärztliches Handeln darstellen. Zusammengefasst werden die klinischen Studien in systematischen Übersichtsarbeiten, die es ermöglichen, die Wirksamkeit von therapeutischen Interventionen, aber auch präventiver und diagnostischer Maßnahmen an einem größeren Patientengut abzuschätzen. Zusammen mit den klinischen Studien stellen die systematischen Übersichtsarbeiten die Grundlagen der EBM dar [5].

\section{EBM: Voraussetzungen}

Damit ein Arzt, als Dermatologe, EBM umsetzen kann, müssen verschiedene Grundvoraussetzungen erfüllt werden.

- Eine Grundvoraussetzung ist die generelle Bereitschaft, sich mit dem Thema aktiv auseinandersetzen zu wollen. Dies wiederum setzt die Bereitschaft voraus, das eigene, zumeist intuitiv geprägte ärztliche Handeln kritisch zu hinterfragen und sich der eigenen Wissenslücken bewusst zu werden [3].

- Ohne EDV-Kenntnisse ist EBM nicht durchführbar. D.h. Grundkenntnisse zum Internet, Suchmaschinen und Medizinischen Datenbanken müssen vorhanden sein bzw. erlernt werden.

- EBM zu praktizieren ist auch nur schwer möglich, wenn die medizinischen Voraussetzungen fehlen. EBM erfordert eine gute medizinische Ausbildung, die neben der Fähigkeit zur genauen Anamneseerhebung und klinischen Untersuchung das Erkennen und Formulieren von klinischen Problemstellungen beinhaltet. Sackett et al. definieren dies als klinische Expertise, die sich aus Sachverstand und Urteilsvermögen zusammensetzt [1].

- Diese vorhandene individuelle klinische Expertise - und dies ist das Herzstück von EBM - muss mit der bestmöglichen wissenschaftlichen Evidenz verknüpft werden mit dem Ziel, einem individuellen, dermatologischen Patienten die beste Behandlung zu ermöglichen. Die bestmögliche vorhandene externe klinische Evidenz stützt sich dabei sowohl auf Forschungsarbeiten aus dem Bereich der patientenorientierten klinischen Forschung als auch auf Arbeiten aus dem Bereich der Grundlagenforschung [1].

\section{EBM: Umsetzung}

Die Umsetzung von EBM wird durch eine strukturierte Vorgehensweise erleichtert. Basierend auf Sackett et al. erfolgt die Umsetzung von EBM in 5 Schritten [6,7]. An erster Stelle steht die Umwandlung der vorhandenen, klinisch relevanten Informatio- nen in eine beantwortbare Frage (I). Anschließend muss mittels verschiedener Suchmethoden (s. u.) die beste externe Evidenz ermittelt(II), deren Güte und Verwendbarkeit kritisch bewertet(III) und dann in die klinische Praxis umgesetzt werden (IV). Zuletzt erfolgt eine zusammenfassende Bewertung des Vorgehens (V).

\section{Anwendung: Stellen einer beantwortbaren Frage}

Die Formulierung einer klinisch relevanten Frage auf der Grundlage eines individuellen Patienten stellt die Basis für das weitere Vorgehen dar. Die Frage sollte strukturiert und im Wesentlichen aus vier Teilen aufgebaut sein. Der erste Teil besteht aus einer genauen Beschreibung des Patienten bzw. seines Problems und erfordert eine vorangehende hinreichende Anamnese und klinische Untersuchung (s.o.). Im zweiten Teil folgt die geplante Intervention, die entweder ein ätiologischer oder prognostischer Faktor, eine diagnostische Untersuchung oder eine Therapie sein kann, im dritten gegebenenfalls eine Vergleichsintervention, im Falle einer Therapie z.B. der Goldstandard bzw. Plazebo. Abschließend sollte die Frage eine für den Patienten und den Arzt relevante klinische Zielgröße enthalten.

Ein Beispiel wäre: Ist bei einer 85-jährigen Patientin mit einer disseminierten Form eines bullösen Pemphigoids (Patient) eine generalisierte topische Glukokortikoidtherapie (Therapie) im Vergleich zur systemischen Glukokortikoidtherapie (Goldstandard) mit einer höheren Remission (Zielgröße) assoziiert?

Wichtig ist zu wissen, dass es nicht nur eine einzige richtige Frage gibt. In fast jeder klinischen Situation sind mehrere Fragen möglich. Dabei sollte diese Frage durchdacht formuliert sein. Eine gut formulierte klinische Frage hat zwei Vorteile. Einerseits erleichtert sie das Finden der Evidenz, andererseits zwingt sie den Kliniker die Patientenpopulation, auf die die Evidenz bezogen werden kann, genauer einzugrenzen und klinische Endpunkte genauer zu definieren. Die Frage „Wie kann ich die Diagnose einer Psoriasis Arthritis am sichersten stellen?" wird sicher zu einer Antwort führen, wobei die Literaturrecherche dazu wahrscheinlich sehr viel Zeit in Anspruch nehmen wird. Hingegen wird eine strukturierte Frage wie „Sind bei einem 28-jährigen Patienten mit Arthritis im Bereich des rechten Knies und umschriebenen, erythematösen Plaques der Streckseiten der Beine Röntgenuntersuchungen im Vergleich zu klinischen Kriterien hilfreich zur sicheren Diagnose einer Psoriasis Arthritis?“ zu genaueren Ergebnissen in kürzerer Zeit führen. Bei der Beschreibung des Patienten ist es daher wichtig, genaue Informationen über die Vorgeschichte, inklusive durchgeführter diagnostischer und therapeutischer Maßnahmen, sowie eine ausführliche klinische Untersuchung einzubeziehen.

Eine gute Frage zu stellen ist nicht leicht, aber erlernbar und hilft beim nächsten Schritt, der Suche nach der besten, externen Evidenz, leichter voranzukommen.

Anwendlung: Suche nach der besten, externen Evidenz

Um Antworten auf eine gut formulierte, strukturierte Frage zu finden, steht eine Vielzahl von Möglichkeiten zur Verfügung. Am wenigsten sind hierfür Lehrbücher geeignet. Diese können 
zwar im Hinblick auf ätiopathogenetische und diagnostische Fragestellungen orientierend zur Seite stehen, im Vergleich zu medizinischen Zeitschriften und elektronischen Datenbanken sind ihre Inhalte bei therapeutischen Fragestellungen zumeist veraltet [9,3]. Zudem spiegeln die meisten Lehrbücher Expertenwissen und damit die niedrigste Stufe der Evidenz wider [2]. Damit wendet sich der Blick auf die Originalliteratur. Die Originalliteratur findet sich in mehr als 20000 medizinischen, davon etwa 200 dermatologischen Zeitschriften. Zusammengefasst werden diese in elektronischen Datenbanken wie z.B. Medline, die monatlich um etwa 33000 Artikel ergänzt wird $[2,10,11]$. Insgesamt wurden in der Medline seit 1966 etwa 11 Millionen Zeitschriftenartikel verschlagwortet. Die Suche nach der besten externen Evidenz erfordert, aus dem unüberschaubaren Angebot die relevanten und besten wissenschaftlichen Arbeiten herauszufiltern.

Neben Medline, Embase und Psychlit steht hier an erster Stelle die nach EBM-Gesichtspunkten geordnete Cochrane Library zur Verfügung [7,12]. Die Cochrane Library beinhaltet im „Cochrane Controlled Clinical Trials Register" (CCTR) gegenwärtig weit über 300000 , im Gegensatz zu den anderen Datenbanken zumeist bewertete Studien, die aus ausführlichen Suchen in den verschiedenen existenten Datenbanken bzw. durch Nachschlagen in Zeitschriften, Konferenzberichten und anderen Quellen, zurück bis ins Jahr 1948, gefunden wurden. Neben den Originalarbeiten werden systematische Übersichtsarbeiten in der „Cochrane Database of Systematic Reviews“ (CDSR) (enthält zur Zeit etwa 1300 Reviews) zusammengefasst. Die Cochrane Library ist mittlerweile in den meisten Universitätskliniken zugänglich. Unter http://www.akh-wien.ac.at/medtools/medlist/databases.htm findet sich zur Zeit (Stand 2/2002) ein kostenloser Zugang zur Cochrane Library über die Gesellschaft der Ärzte in Wien, im Internet unter http://www.billrothhaus.at.

Innerhalb des Cochrane Networks ist die Dermatologie in der Cochrane Skin Group vertreten. Aufgabe der Cochrane Skin Group (Zugang über: http://www.cochrane.de) ist die Förderung und Durchführung von systematischen Übersichtsarbeiten zu dermatologischen und allergologischen Erkrankungen.

Bei der nun folgenden Literaturrecherche in einer solchen Datenbank zeigt sich die Schwierigkeit beispielhaft darin, dass in einer Medline-Suche nur 31 von 73 aller in der Zeitschrift Clinical and Experimental Dermatology veröffentlichten RCTs, die zuvor per Hand herausgesucht wurden, identifiziert werden konnten [13]. Grund dafür ist eine mangelhafte Verschlagwortung von klinischen Studien [10]. Deswegen sollte man, wenn immer möglich, auf Datenbanken mit einer besseren Indexierung, wie die der Cochrane Collaboration, zurückgreifen [7,12].

Zur Orientierung und Weiterbildung stehen neben diesen medizinischen Datenbanken auch nach EBM-Gesichtspunkten konzipierte medizinische Zeitschriften zur Verfügung. Hierzu gehört z.B. das American College of Physicians (APC) Journal sowie andere Zeitschriften zur Evidenzbasierten Medizin [3,14,15]. Das ACP Journal (http://www.acpjc.org/) beispielsweise wird seit 1991 sechsmal im Jahr vom American College of Physicians veröffentlicht. Jeder darin erscheinende Artikel wird nach strengen Evidenzkriterien ausgewählt und informiert in strukturierten Zusammenfassungen und einem Kommentar über neue Studien- ergebnisse der Inneren Medizin. Seit 1995 gibt es als weiteres Journal die Zeitschrift „Evidence based Medicine“, welche in ähnlicher Weise relevante Studien der Inneren Medizin, Neurologie, Chirurgie, Geburtshilfe und Gynäkologie sowie Pädiatrie in strukturierter Form bespricht und kommentiert. Beide Zeitschriften sind ausgezeichnete Quellen zur raschen Orientierung über wichtige, qualitativ hochstehende Forschungsergebnisse zu Diagnostik, Prognose, Therapie und gesundheitsökonomischen Untersuchungen [3], enthalten allerdings wenige bzw. keine Informationen zu dermatologischen Erkrankungen. Neuerdings gibt es innerhalb der dermatologischen Zeitschriften erste Ansätze, EBM zu integrieren. So erscheint z. B. in den „Archives of Dermatology“ eine eigene Sektion zu EBM in der Dermatologie.

Ist die Literatur gefunden, muss diese im nächsten Schritt kritisch evaluiert werden.

\section{Anwendung: Kritische Beurteilung der gefundenen Evidenz}

Die Evaluierung der gefunden Literatur erfolgt nach den Regeln eines guten Journalclubs, der bedauerlicherweise an den wenigsten Kliniken zu finden ist, so dass der kritische Umgang mit der Literatur erst erlernt werden muss („How to read a paper“). Hilfreich ist dabei eine grobe Evidenzhierarchie, die es ermöglicht, anhand der Struktur einer Studie einen ersten Überblick über deren Güte zu gewinnen. Beispielhaft sei hier die Evidenzhierarchie für therapeutische Interventionen, modifiziert nach dem „Oxford Center for Evidence based Medicine“ [16,17], vorgestellt (Tab.1).

Systematische Übersichtsarbeiten beinhalten - wann immer möglich - Metaanalysen vergleichbarer Arbeiten. Der Vorteil dieser Methode besteht darin, gerade bei mehreren klinischen Studien mit kleinen Stichprobenumfängen, diese mittels quantitativ statistischer Methoden zu kombinieren, um dann, durch die erhöhte Studienstärke, klinisch relevante Ergebnisse zu erhalten. Die Metaanalyse ist allerdings nicht vor irreführenden Schlussfolgerungen gefeit, da negative Studien weniger oft publiziert werden als Studien mit positiven Ergebnissen (Publication Bias) $[3,18]$. Aufgrund dessen sollte man eine Metaanalyse immer genau prüfen, ob und welche Anstrengungen zur Identifikation von unpublizierten Studien unternommen wurden [3,19]. Neben systematischen Reviews bieten größere randomisierte, klinisch kontrollierte Studien (RCT) die beste klinische Evidenz [5,20,21],

Tab. 1 Evidenzhierarchie

\begin{tabular}{cl}
\hline Stufe & Evidenz-Typ \\
\hline la & $\begin{array}{l}\text { wenigstens ein systematischer Review auf der Basis methodisch } \\
\text { guter, randomisierter, klinisch kontrollierter Studien (RCTs) }\end{array}$ \\
Ib & $\begin{array}{l}\text { wenigstens eine ausreichend große, methodisch gute, } \\
\text { randomisierte, klinisch kontrollierte Studie }\end{array}$ \\
Ila & $\begin{array}{l}\text { wenigstens eine gute klinische Studie ohne Randomisierung, mit } \\
\text { adäquatem Design }\end{array}$ \\
IIb & $\begin{array}{l}\text { wenigstens eine gute Studie eines anderen Typs - eine } \\
\text { quasi-experimentelle Studie }\end{array}$ \\
III & $\begin{array}{l}\text { mehr als eine methodisch gute nichtexperimentelle Studie } \\
\text { IV }\end{array}$ \\
& $\begin{array}{l}\text { Meinungen und Überzeugungen von klinischen Experten; } \\
\text { Expertenkommissionen; Fallberichte }\end{array}$
\end{tabular}


so dass sie heute, sofern sie gemäß definierter Qualitätskriterien durchgeführt wurden, den Goldstandard der EBM darstellen. Auf der nächsten Stufe in der Evidenzhierarchie finden wir Beobachtungsstudien, die in einzelnen Fällen gleiche Ergebnisse liefern wie RCT [5,8], sogar in seltenen Fällen überlegen sein können [5]. Allerdings sollten zuerst die Artikel identifiziert werden, die in der Evidenzhierarchie an oberster Stelle stehen, und nur beim Fehlen dieser Studien auf solche mit niedrigerer Evidenz zurückgegriffen werden [5].

\section{Anwendung: Umsetzung in die klinische Praxis}

Nach Sichtung und Validierung der vorhandenen Evidenz sollten, vor der Umsetzung in z. B. Diagnostik und Therapie, die Ergebnisse noch einmal kritisch hinterfragt werden. Dabei ergeben sich unterschiedliche Ansatzpunkte:

- Ist der Patient mit den in verschiedenen Studien Untersuchten vergleichbar [2]? D.h. können wir Ergebnisse aus einer Studie an ansonsten gesunden, 30- bis 50-jährigen Psoriasispatienten (Studienpopulation) auf einen 15-jährigen oder einen 70-jährigen multimorbiden Patienten übertragen?

- Ist die Zielgröße klinisch sinnvoll?, d.h. z.B. eine Verminderung betroffener Hautoberfläche von $15 \%$ auf $10 \%$ [2]. Ist der Therapieerfolg für den Patienten überhaupt sichtbar und wird der Patient zufriedengestellt?

- Sind die Daten richtig interpretiert worden? Hier ist Vorsicht v.a. bei der falschen Übertragung von für Gruppen geltenden Daten auf einzelne Patienten angebracht [22,2]. Das Ergebnis einer Studie, dass 80\% der Patienten 6 Monate unter einer bestimmten Therapie rezidivfrei sind, bedeutet nicht, dass dieser bestimmte Patient mit einer Wahrscheinlichkeit von $80 \%$ rezidivfrei ist, sondern dass in einer Gruppe von Patienten mit ähnlichen Eigenschaften wahrscheinlich 80\% der Behandelten rezidivfrei sind [2]. Insgesamt bedeutet dies, dass für den einzelnen Patienten keine Aussage möglich ist.

- Mit welchen Nebenwirkungen ist zu rechnen, und wie steht das Verhältnis der Nebenwirkungen zum erwarteten Benefit?

All dies sind Fragen, die man sich beantworten sollte, bevor es zu einer Umsetzung in die Praxis kommt. Anschließend erfolgt schließlich der fünfte und letzte Punkt, die Validierung der Vorgehensweise.

\section{Anwendung: Validierung der Vorgehensweise}

EBM kann nur funktionieren, wenn die einzelnen Schritte der Entscheidungsfindung und die Entscheidung selbst danach kritisch hinterfragt werden. Über die Evaluierung aller stattgehabten Schritte und Prozeduren hinaus stellt sich hier die Frage, ob durch die Anwendung von EBM selbst, im Vergleich zum ursprünglichen Vorgehen, eine Verbesserung der Situation für Arzt und Patient stattgefunden hat. Denn auch EBM muss sich einer wissenschaftlichen Evaluierung stellen.

\section{Abgrenzung der EBM zu Leitlinien}

Abgegrenzt werden muss EBM von Leitlinien, wobei Leitlinien evidenzbasiert sein können. Letztere sind laut Ärztlicher Zentral- stelle Qualitätssicherung (AZQ) und Arbeitsgemeinschaft der Wissenschaftlichen Medizinischen Fachgesellschaften (AWMF) systematisch entwickelte Entscheidungshilfen über die angemessene ärztliche Versorgung bei speziellen gesundheitlichen Problemen [23]. Leitlinien dienen nur als Orientierungshilfen, die Handlungs- oder Entscheidungskorridore darstellen, von denen in begründeten Fällen abgewichen werden kann oder sogar muss. Sie beziehen sich auf die Behandlung einer Krankheit an sich und nicht, wie die EBM, auf die Behandlung von Patienten mit ganz bestimmten Merkmalen beziehungsweise individuellen Patienten (laut Definition). Mit Hilfe der Leitlinien ist es möglich, die zunehmende Informationsmenge an wissenschaftlicher Evidenz sowie Expertenmeinung über „gute medizinische Praxis" den Leistungsträgern im Gesundheitswesen zu vermitteln. Gemeinsamkeit zwischen beiden besteht jedoch darin, dass die Evidenzbasierte Medizin genauso wenig vorschreibt, was in der Behandlung von Patienten zu tun ist.

\section{Synopsis}

EBM ist kein statischer, sondern ein sich kontinuierlich weiterentwickelnder Prozess in der Medizin, der sich in der Dermatologie zur Zeit noch am Anfang seiner Entwicklung befindet. Auf Dauer werden sich die wenigsten Mediziner dieser Vorgehensweise entziehen können. Hier liegt es an jedem Einzelnen, seine Bereitschaft zu zeigen, aktiv an der Umsetzung von EBM teilzunehmen. Wichtig ist es, die Voraussetzungen, d.h. die Strukturen, die eine Umsetzung erleichtern, zu verbessern, so dass immer mehr Ärzte und auch Nicht-Ärzte Zugang dazu haben werden.

Auf Dauer ist durch die konsequente Umsetzung von Evidenzbasierter Medizin eine Verbesserung der Versorgungssituation der Patienten zu erwarten. Ärztliche Tätigkeit wird einer zunehmenden Qualitätssicherung unterzogen, was beiden Seiten, der Arztund Patientenseite, zugute kommt. Sozusagen ad hoc können neueste wissenschaftliche Erkenntnisse in den Behandlungsalltag miteinbezogen werden.

$\mathrm{Zu}$ beachten gilt allerdings, dass gefundene Inhalte nicht immer zwangsläufig auf den einzelnen Patienten übertragbar sind. EBM ist, und dies sollte hier noch einmal explizit gesagt werden, keine Kochbuchmedizin [1,2]. Nicht immer lassen sich komplexe medizinische Sachverhalte auf eine einzelne Frage reduzieren. Hier bedarf es der verantwortlichen Umsetzung des vorhandenen und standardisiert evaluierten Wissens unter Einschluss der klinischen Expertise und der Bedürfnisse des Patienten.

\section{Literatur}

${ }^{1}$ Sackett DL, Rosenberg WM, Gray JA, Haynes RB, Richardson WS. Evidence based medicine: what it is and what it isn't. BMJ 1996; 312: $71-72$

2 Williams H. Evidence based dermatology - a bridge to far. $\mathrm{Cl}$ and Exp Dermatol 2001; 26 (8): 714 - 724

${ }^{3}$ Bucher HC, Egger M, Schmidt JG, Antes G, Lengeler CH. Evidencebased medicine: approach to a more rational medicine. Arbeitsgemeinschaft Cochrane Collaboration Schweiz Schweiz Rundsch Med Prax 1997; 86: 606-612

${ }^{4}$ Schloemer G. Evidence-based nursing: a method for nursing practice? Pflege 2000; 13: 47-52 
${ }^{5}$ Barton S. Which clinical studies provide the best evidence? The best RCT still trumps the best observational study. BMJ 2000; 321: $255-256$

${ }^{6}$ Sacket DL, Richardson WS, Rosenberg W, Haynes RB. Evidence-based Medicine - How to practice and teach EBM. Churchill Livingstone, 1996

${ }^{7}$ Bigby M. Evidence-based medicine in dermatology. Dermatol Clin 2000; 18: $261-276$

${ }^{8}$ Benson K, Hartz AJ. A comparison of observational and randomized controlled trials. N Engl J Med 2000; 342: 1878-1886

${ }^{9}$ Antmann EM, Lau J, Kupelnick B, Mosteller F, Chalmers TC. A comparison of results of metaanalyses of randomized control trials and recommendations of clinical experts. JAMA 1992; 268: 240-248

${ }^{10}$ Delamere FM, Williams HC. How can hand searching the dermatological literature help people with skin problems? Arch Dermatol 2001; 137: $332-335$

${ }^{11}$ Koneczny N. Evidence.de - Internetbasierte medizinische Leitlinien: Ein zukunftsfähiges Modell. Deutsches Ärzteblatt 1998; 36: 22

${ }^{12}$ Williams H, Adetugbo K, Po AL, Naldi L, Diepgen T, Murrele D. The Cochrane Skin group. Preparing, maintaining, and disseminating systematic reviews of clinical interventions in dermatology. Arch 1998; 134: $1620-1626$

${ }^{13}$ Adetugbo K, Williams $\mathrm{H}$. How well are randomized controlled trials reported in the dermatology literature. Arch Dermatol 2000; 136: $381-385$

14 Davidoff F, Haynes B, Sackett D, Smith R. Evidence based medicine. BMJ 1995; 310: 1085-1086

${ }^{15}$ Haynes RB. The origins and aspirations of ACP Journal Club. ACP Journal Club 1991; 114: A18

${ }^{16}$ Canadian Task Force on the Periodic Health Examination. The periodic health examination. CMAJ 1979; 121: $1193-1254$

${ }^{17}$ Canadian Task Force on the Periodic Health Examination. The Canadian guide to clinical preventive health care. Ottawa: 1994

${ }^{18}$ Egger M, Davey Smith G. Misleading meta-analysis. BMJ 1995; 310: $752-754$

${ }^{19}$ Arbeitsgemeinschaft Cochrane Collaboration. Die Cochrane Collaboration. Schweiz Arztezeitung 1996; 77: $117-120$

${ }^{20}$ Ziegler S, Luhmann D, Raspe H, Windeler J. Rapid reviews for evidence-based decision support. (Restricting) requirements. Z Arztl Fortbild Qualitatssich 2001; 95: 105-111

${ }^{21}$ Guyatt GH, Haynes RB, Jaeschke RZ, Cook DJ, Green L, Naylor CD, Wilson MC, Richardson WS. User's Guides to the Medical Literature: XXV. Evidence-based medicine: principles for applying the User's Guides to patient care. Evidence-Based Medicine Working Group. JAMA 2000; 284: 3128 - 3128

22 Bakan D. The general and the aggregate: a methodological distinction. Perceptual Motor Skills 1955; 5: 211 - 212

${ }^{23}$ Arbeitsgemeinschaft der Wissenschaftlichen Medizinischen Fachgesellschaften, Ärztliche Zentralstelle Qualitätssicherung. Das Leitlinien-Manual: Entwicklung und Implementierung von Leitlinien in der Medizin. ZaeFQ 2001; 95 (Suppl. 1): 5-8

\section{Buchbesprechung}

\section{Sexualmedizin. Grundlagen und Praxis}

Beier, K. M., H. A. Bosinski, U. Hartmann, K. Loewit

574 Seiten, 73 Abb. Urban \& Fischer, München 2001.

Geb. 84,95. ISBN 3-437-51086-X

Die Sexualmedizin hatte schon immer viele Berührungspunkte mit dem Fach der Dermato-Venerologie. Andrologie, STD und Geschlechtskrankheiten sind integraler Bestandteil unseres Faches gewesen. Leider werden sie immer mehr mangels Interesse oder Unkenntnis vernachlässigt. Im Rahmen seiner Tätigkeit wird jedoch der Hautarzt immer wieder mit sexuellen Problemen des Patienten konfrontiert. Es ist das Verdienst der vier Autoren unter Mitarbeit einiger Kollegen, u. a. des Dermatologen H. J. Vogt, München, hier erstmals ein umfassendes, modernes Werk verfasst zu haben, welches diesen wichtigen Bereich menschlicher Biologie und Psyche abhandelt. So werden die Grundlagen der Sexualmedizin und der entsprechenden Anthropologie beschrieben. Es folgen die Praxis der Sexualmedizin mit Hinweisen auf Befunde und deren Behandlung, Nosologie und Epidemiologie sexueller Funktionsstörungen sowie sexuelle Funktionsstörungen der Frau und des Mannes und deren Behandlung. Weitere Kapitel befassen sich mit Geschlechtsidentitätsstörungen, Paraphilien, Sexualdeliquenz und Opfer sexueller Übergriffe. Krankheits- und behandlungsbedingte Sexualstörungen. Schließlich Sexualerziehung und Kontrazeptionsberatung, gestörte Sexualität bei Infertilität und Sterilität, STD und HIV/ AIDS. Gesetzestexte, Praxisleitlinien und ein Literaturverzeichnis vervollständigen den Text. Großer Wert wird auf das Gespräch, die Patientenführung, Diagnostik, Beratung und Therapie gelegt. Insgesamt ein ausführliches und eingehendes Werk über die Sexualmedizin welches jeder Dermatologe intensiv studieren sollte.

G. Brehm, Ludwigshafen 